\title{
Análisis de la metalurgia y mecánica de la fractura en la ZAC en un proceso por arco sumergido ${ }^{(\cdot)}$
}

\author{
R. González ${ }^{(*)}$ y F. Carrillo ${ }^{(* *)}$
}

\begin{abstract}
Resumen Los aceros microaleados de alto límite elástico, grupo al que pertenecen los aceros de diseño 450 EMZ del presente trabajo, son aceros utilizados en plataformas offshore y estructuras sometidas a extremas condiciones de trabajo, a las que se les requiere alta resiliencia y tenacidad a la fractura a bajas temperaturas. Estos aceros son sometidos a un control en sus parámetros de soldadura a fin de recibir una baja energía calorífica, que permitan mantener unos valores de tenacidad adecuados en su zona afectada por el calor (ZAC). En este trabajo se pretenden analizar los valores obtenidos de dureza, resiliencia y tenacidad, así como los puntos de mayor fragilidad de la ZAC (1).
\end{abstract}

Palabras clave: Aceros microaleados. Soldadura por arco sumergido. Tenacidad de la zona afectada.

\section{Analysis of the metallurgy and mechanics of the fracture in the $\mathrm{HAZ}$ in a submerged arc process}

\begin{abstract}
High tensile, microalloyed steels, such as 450 EMZ design steels which are studied in this article, are used in offshore platforms and other structures subject to extreme working conditions, requiring high resilience and fracture toughness at low temperatures. These steels have been subject to a control in their welding parameters to obtain a low heat input which allows for the maintenance of correct toughness values in their heat affected zone (HAZ). The present article analyses the values of hardness, resilience and toughness, as well as the points of greatest brittleness of the HAZ (1).
\end{abstract}

Keywords: Microalloyed steels. SAW welding. Heat affected zone.

\section{METALURGIA DE LA ZAC}

Las probetas objeto de este estudio se obtienen del cupón soldado en arco sumergido (S.A.W.), denominado 1-1G HAZ. A efectos de los productos de transformación que se producen en el enfriamiento dentro de la zona afectada por el calor ZAC, constatamos que con una energía aportada de 2,3 $\mathrm{kJ} / \mathrm{mm}$ se obtiene una temperatura de pico de fusión de $1.527^{\circ} \mathrm{C}$, un tiempo de enfriamiento $\Delta t_{8 / 5}$, de 10 segundos, prácticamente constante para toda la ZAC, según muestra el ciclo teórico de Rosenthal para este proceso, placa gruesa y características físicas del acero microaleado $450 \mathrm{EMZ}$. En la figura 1 se ha representado la curva C.C.T. (Continuous

$(\bullet$ Trabajo recibido el día 5 de octubre de 1998 y aceptado en su forma final el día 20 de mayo de 1999.

(*) Dpto. de Ingeniería Mecánica. Universidad de Cádiz.

(**) Dpto. de Construcciones Navales. Universidad de Cádiz.
Cooling Transformation) para los aceros microaleados utilizados en Off-shore, como es el caso de los $450 \mathrm{EMZ}(1)$.

\section{ESTUDIO DE LA TENACIDAD A LA FRACTURA DE LA ZAC. DISCUSIÓN DE LOS RESULTADOS}

\subsection{Ensayo de CTOD}

El ensayo de mecánica de fractura se llevó a cabo sobre el panel antes numerado 1-1G HAZ en la ZAC, al que pertenecen las probetas P1S, P1T, $\mathrm{P} 1 \mathrm{~A}, \mathrm{P} 1 \mathrm{~B}$ y P1C en las que hemos llevado a cabo el presente estudio.

Dicho panel, de $75 \mathrm{~mm}$ de espesor, $980 \mathrm{~mm}$ de longitud de soldadura y $750 \mathrm{~mm}$ de ancho, se ensayó en condiciones "As welded" (sin tratamiento 


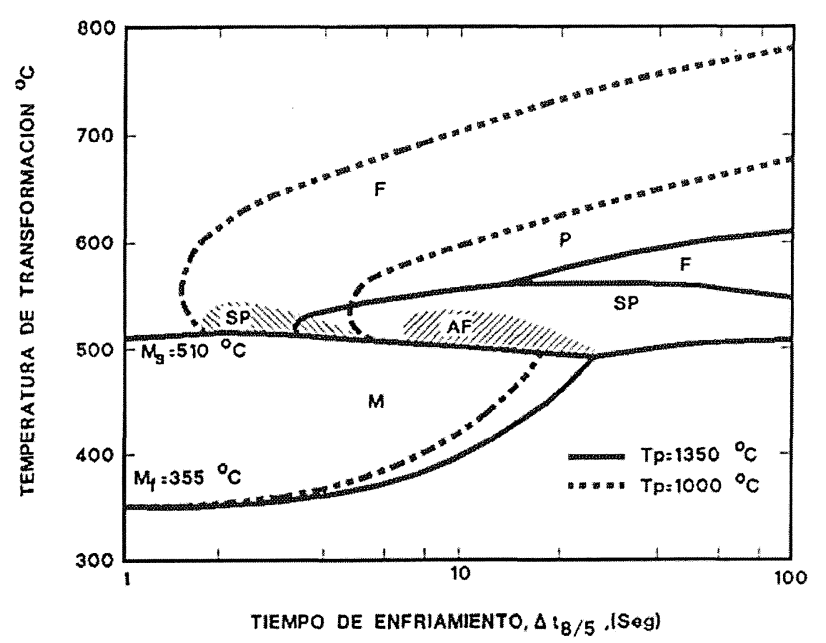

FIG. 1.- Diagrama CCT para aceros microaleados, según Akselsen Institute of Technology Trondheim (1).

FIG. 1.- CCT diagram for microalloy steels, in accordance with Akselsen Institute of Technology of

Trondheim (1).

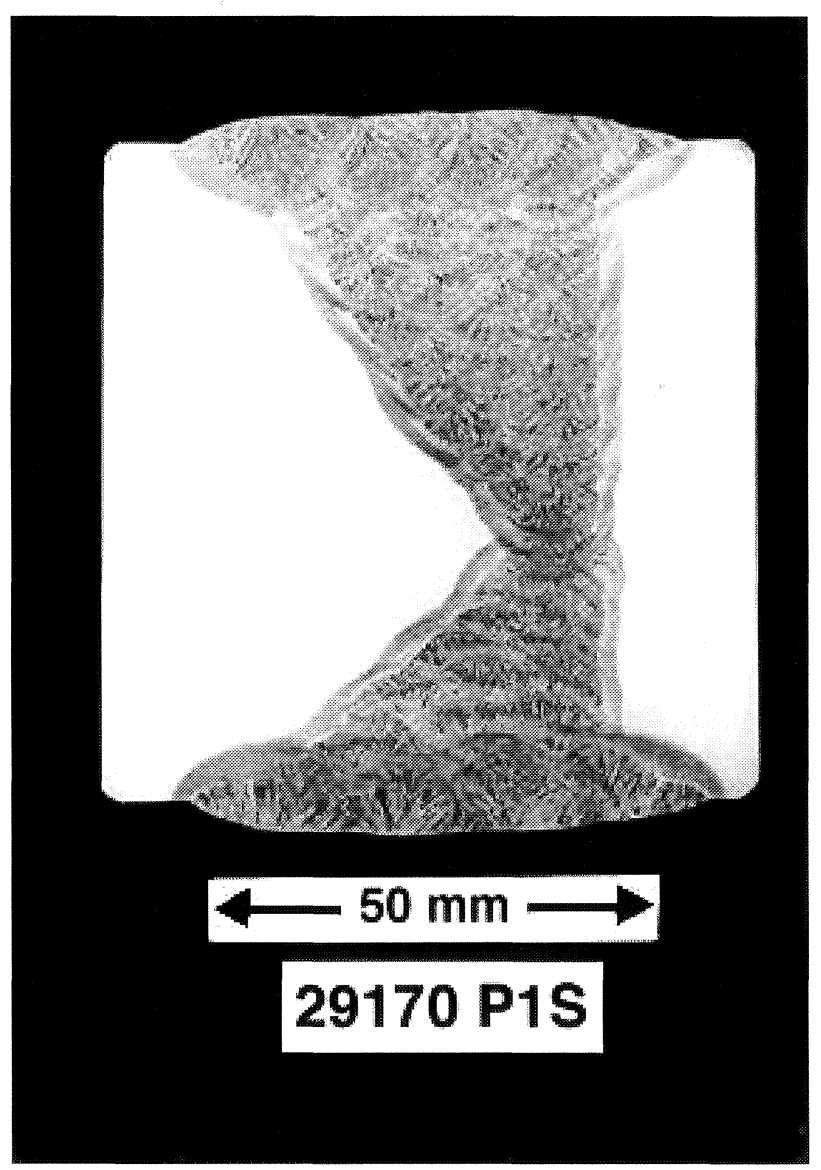

FIG. 2.- Macrografía óptica de la sección tomada por el inicio de la soldadura.

FIG. 2.- Optical macrograph taken from welding start. térmico post-soldeo) y se utilizó para ensayar la ZAC. La soldadura fue realizada en $\mathrm{K}$, en un proceso de arco sumergido (SAW), con un hilo Autrod 13,24 y flux OK 10,62, debidamente homologado, para soldar el metal base BS 7191450 EMZ tipo 2. La probeta P1S, cuya macrografía está representada en la figura 2 y que es objeto del presente estudio, se obtuvo a $50 \mathrm{~mm}$ del extremo del panel, al comienzo de la soldadura, extrayendo una muestra que fue atacada con $2 \%$ de Nital y pulida con pasta de diamante de $1 \mu \mathrm{m}$.

De la macrografía correspondiente a la probeta P1T (Fig. 3) se obtuvo un pre-estudio metalográfico en el que se valoró el tamaño de grano, conforme a ASTM E 112 (2), siguiendo el método de Heyn en los dos tercios del espesor de la probeta, obteniéndose los resultados que muestran las tablas I y II. Como vemos, se observa un elevado porcentaje de tamaño de grano fino en la ZAC, debido al control de la energía aportada y al número de pasadas con el que ha sido realizada la soldadura.

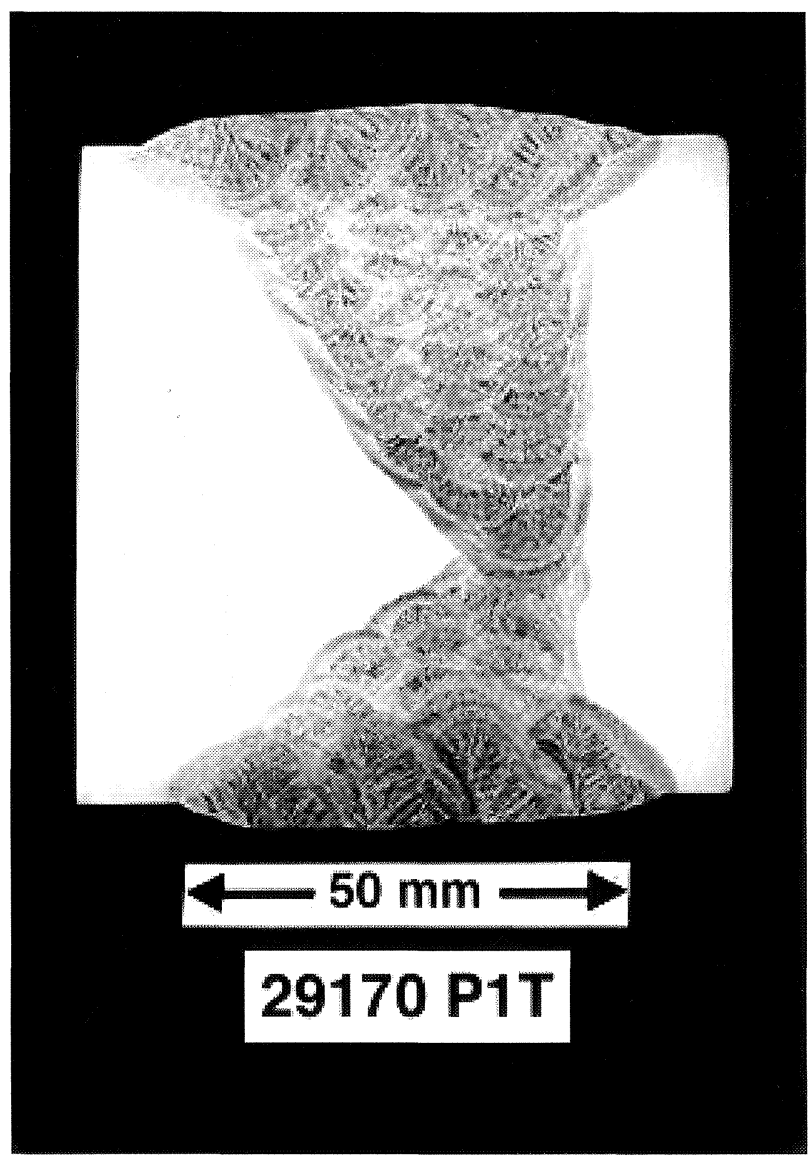

FIG. 3.- Macrografía óptica de la sección tomada por el final de la soldadura.

FIG. 3.- Optical macrograph taken from one end welding. 
TABLA I.- Valoración del tamaño de grano sobre probetas de CTOD (P1S, P1T) prefisuradas TABLE I.-Results of gran size assessment along CTOD specimens (PIS, PIT) precrack

\begin{tabular}{|c|c|c|c|}
\hline Categoría del tamaño de grano según ASTM E112 & $<5$ & $5-7$ & $>7$ \\
\hline Comienzo de la soldadura P1S & $12,5 \%$ & $48,2 \%$ & $39,3 \%$ \\
Fin de la soldadura P1T & $4,2 \%$ & $46,6 \%$ & $49,2 \%$ \\
\hline
\end{tabular}

* Muestras tomadas a $50 \mathrm{~mm}$ de los finales de soldadura

** Porcentaje sobre los $2 / 3$ centrales del espesor

TABLA II.- Valoración del tamaño de grano sobre las probetas de CTOD (P1S, P1B, P1C) prefisuradas

TABLE II.-Results of grain assessment along CTOD precracked specimens (PIS, P1B, PIC)

\begin{tabular}{|c|c|c|c|c|}
\hline \multicolumn{2}{|c|}{$\begin{array}{c}\text { Categoría del tamaño de grano de la ZAC } \\
\text { según ASTM E112 }\end{array}$} & $<5$ & $5-7$ & $>7$ \\
\hline Probeta & Metal base & & & \\
P1A & $0,0 \%$ & $0,0 \%$ & $12,7 \%$ & $87,3 \%$ \\
P1B & $61,9 \%$ & $0,0 \%$ & $6,9 \%$ & $31,2 \%$ \\
P1C & $56,8 \%$ & $0,0 \%$ & $4,2 \%$ & $39,0 \%$ \\
\hline
\end{tabular}

* Porcentaje sobre los $2 / 3$ centrales del espesor

En el gráfico de la figura 4, se han representado los valores de CTOD en función del tamaño de grano. A mayor porcentaje del WM (metal de soldadura) los valores de CTOD decrecieron, lo que indica una mayor fragilidad del metal aportado en las zonas próximas a LF (línea de fusión). No se encontró en esta zona el grano grueso de tamaño < 5 ASTM. En cuanto al tamaño entre 5 y 7 ASTM, se observa una tendencia a aumentar el CTOD conforme el tamaño de grano disminuye. Para tamaño de grano fino ( $>7$ ASTM) la tendencia es también creciente y estos resultados confirman el aumento de la tenacidad para este tamaño de grano. En los tamaños de grano que se muestran en las tablas I y II, se observa un alto porcentaje de grano fino debido a las múltiples pasadas de esa soldadura y a la energía aportada $(2,4 \mathrm{~kJ} / \mathrm{mm})$.

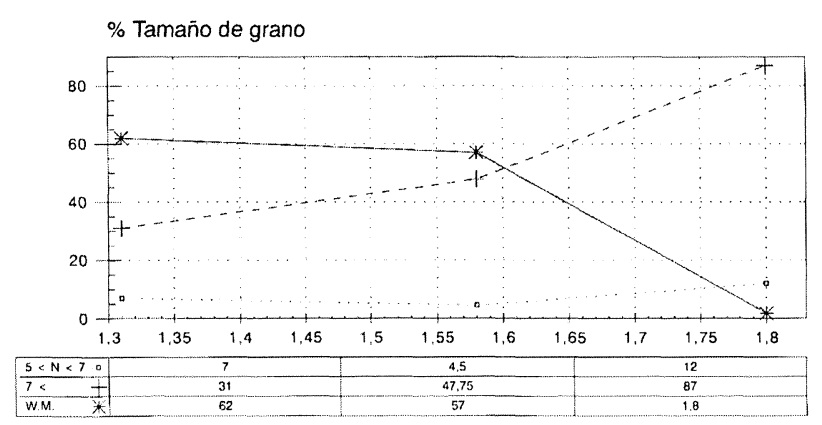

Fig. 4.- Tamaño de grano - CTOD. Proceso SAW.

FIG. 4.-Grain size-CTOD. Process SAW.
El ensayo de fractura fue llevado a cabo por doblado en 3 puntos y entalla sobre el centro de la probeta (SENB3), elaborada conforme la norma BS 5762 (3) y según la geometría preferida $\mathrm{B} \times 2 \mathrm{~B}$ siendo su espesor $B=75 \mathrm{~mm}$ (Fig. 5). El procedimiento de construcción y marcado de las probetas se hizo según la norma EEMUA 2.2.2 (4) y la entalla en dirección T.L. (Fig. 6).

La probeta se comprimió, inicialmente, a 0,005 B por cada lado, a fin de evitar las tensiones residuales y obtener un perfil de prefisurado uniforme.

Los ensayos se llevaron a cabo a $-10{ }^{\circ} \mathrm{C}$ conforme las normas BS 5762. Para la determinación del valor de la fractura se tomó la media de los valores del límite elástico del metal base y metal aportado, corregidos mediante la siguiente fórmula, al valor de $-10{ }^{\circ} \mathrm{C}$.

$$
\begin{gathered}
\sigma_{\mathrm{ys}}=\left(\frac{\sigma_{\mathrm{ymb}}+\sigma_{\mathrm{yma}}}{2}\right)+ \\
+\frac{10^{5}}{491+1,8 T}-189(\mathrm{MPa})
\end{gathered}
$$

Donde: $\sigma_{\mathrm{ymb}}=$ Límite elástico del metal base.

$\sigma_{\text {yma }}=$ Límite elástico del metal de aportación.

$T=$ Temperatura CTOD $=-10^{\circ} \mathrm{C}$.

El límite elástico del material dado por el fabricante fue de $434 \mathrm{~N} / \mathrm{mm}^{2}$, el del metal de soldadura 


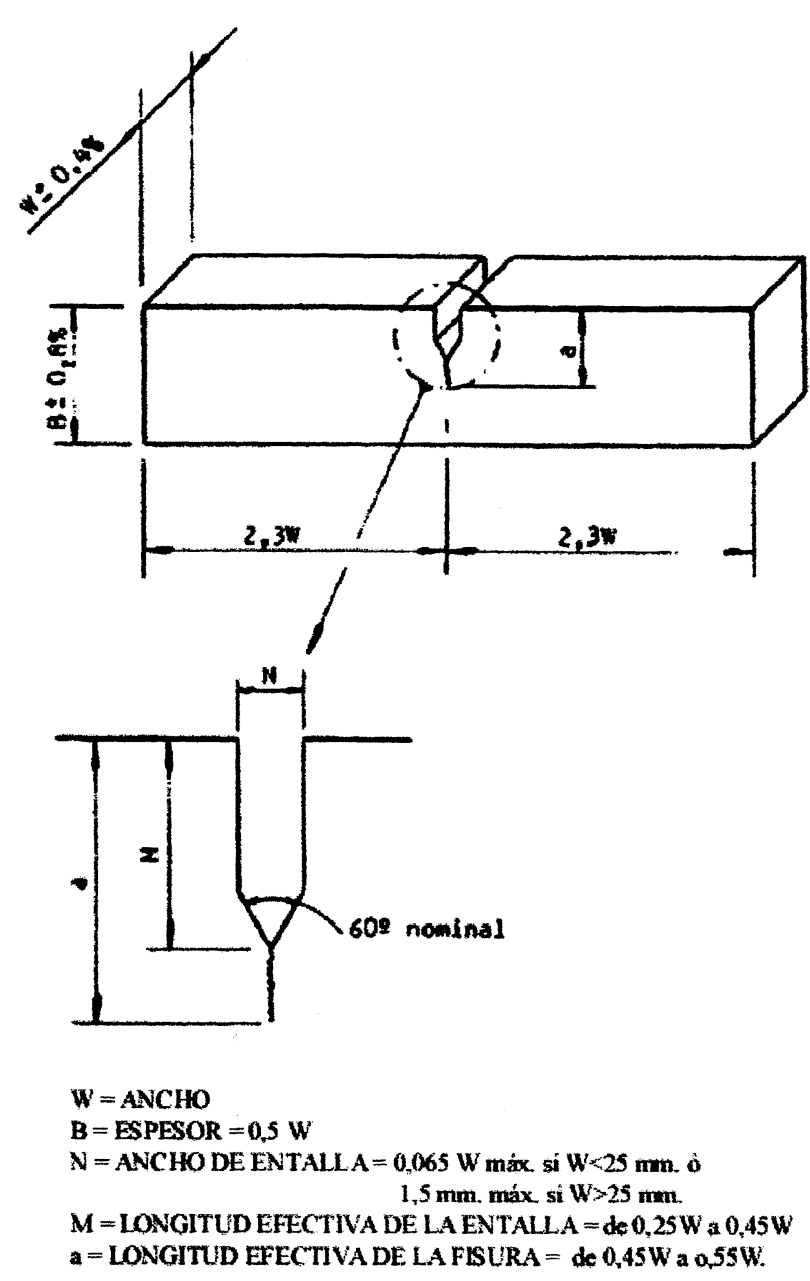

FIG. 5.- Probeta CTOD.

FIG. 5.- CTOD Specimen.

de $609 \mathrm{~N} / \mathrm{mm}^{2}$, obtenidos por ensayo de tracción. Introduciendo estos valores en la ecuación [1] se obtiene el resultado de $544 \mathrm{~N} / \mathrm{mm}^{2}$. Los valores obtenidos para los CTOD, $\delta$ y factor de intensidad de tensiones $K$ se dan en la tabla III.

Los resultados obtenidos para el CTOD fueron bastante aceptables y superiores a los requeridos por la norma BS 5762 que exige un mínimo de $\delta=$ $0,2 \mathrm{~mm}$.

Los valores correspondientes al diagrama de carga desplazamiento para las probetas P1A, P1B,

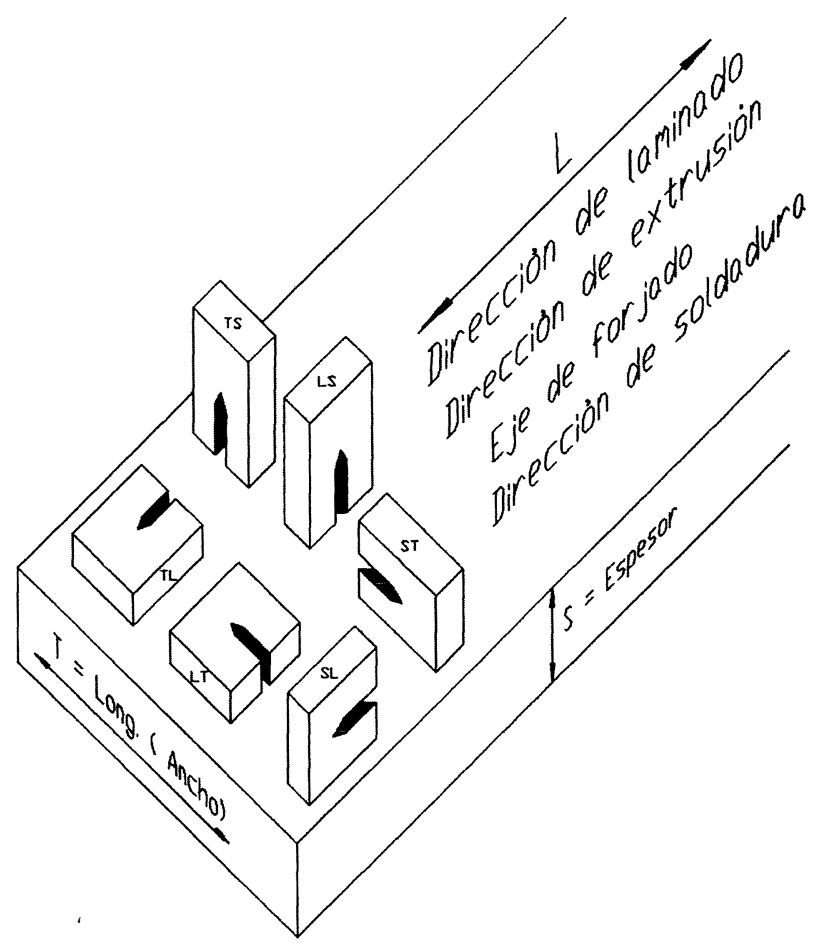

FIG. 6.-Designación de las distintas orientaciones.

FIG. 6.-Designation of the varions orientations.

P1C, obtenidos de la máquina de ensayo se reflejan en las figuras 7 y 8.

Para el estudio metalográfico se obtuvieron rebanadas de $15 \mathrm{~mm}$ de las probetas ensayadas paralelas a la fractura, como se indica en la figura 9, con el objeto de obtener la superficie de la cara de la fractura.

Se resaltan los altos valores de tenacidad a la fractura, $K\left(\mathrm{~N} / \mathrm{mm}^{3 / 2}\right)$ obtenidos. Si a estos valores de tenacidad les aplicamos la ecuación de Robert y Newton (6) obtenemos valores en torno a $156 \mathrm{~J}<$ $\mathrm{CVN}<170 \mathrm{~J}$, que se aproximan a la resiliencia para $-40{ }^{\circ} \mathrm{C}$ en la raíz LF y $-30{ }^{\circ} \mathrm{C}$, en los cordones superiores LF y temperatura próxima a la temperatura de transición de este material, lo que cumple razonablemente con la expresión empírica de Robert y Newton. Se observa que la probeta P1A, que tuvo una fractura dúctil, tiene un porcentaje de grano fino elevado ( $>7$ ASTM), es decir, 87,3 \% (Tabla II).

TABLA III.- CTOD- Tenacidad a la fractura.

TABLE III._-Fracture toughness test results.

\begin{tabular}{|c|c|c|c|}
\hline Probeta & $K\left(\mathrm{~N} / \mathrm{mm}^{3 / 2}\right)$ & C.T.O.D. $(\delta)$ & Tipo de resultado conforme BS 5762 \\
\hline P1A & 6.565 & 1,80 & Ensayo parado \\
P1B & 6.404 & 1,31 & $\delta_{\mathrm{u}}$ último valor \\
P1C & 6.750 & 1,58 & $\delta_{u}$ último valor \\
\hline
\end{tabular}




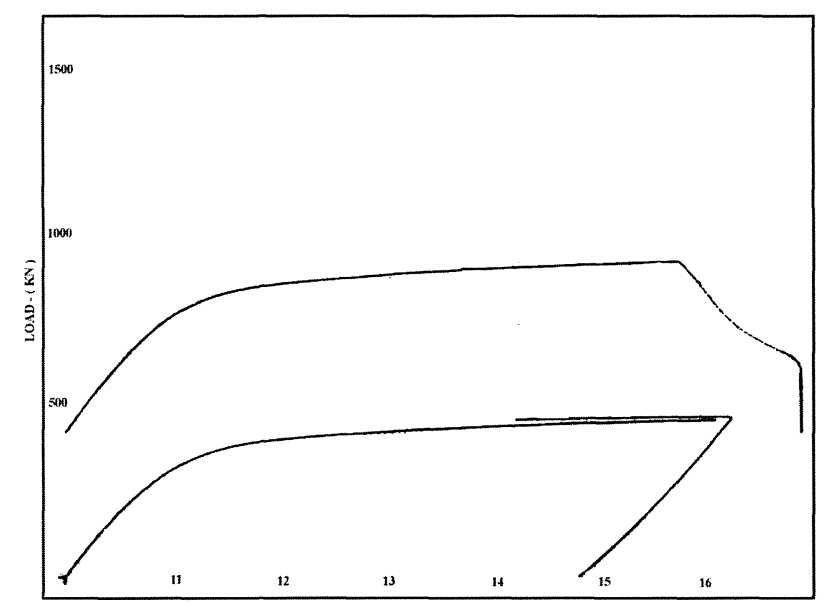

FIG. 7.- Registro de máquina de ensayo CTOD P1A-P1B.

FIG. 7.- CTOD results registered in the testing machine PIA-PIB.

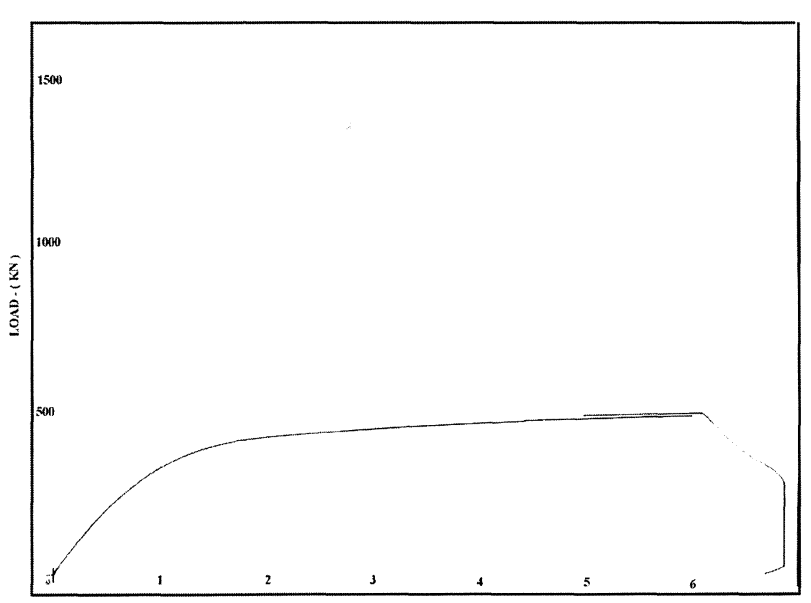

FIG. 8.- Registro máquina de ensayo CTOD P1C.

FIG. 8.- CTOD results registered in the testing machine PIC.

Referente a la probeta P1B, que mostró fractura frágil, se obtuvo una segunda sección en el comienzo de la grieta cuya macrografía se da en la figura 10 , donde se indica, ampliada al microscopio óptico, el comienzo de la fractura en la zona intercrítica de la ZAC (Figs. 10 y 11). La fisura se midió en su comienzo a 3,3 mm de LF (región intercrítica, tabla IV). Asimismo, observando el diagrama de dureza de la probeta P1T (Fig. 12) tomada a los $50 \mathrm{~mm}$ finales del panel soldado, la dureza máxima en la zona de raíz a esta distancia de LF es de 223 HV 10. La razón de la fragilización de una zona intercrítica, a pesar de ser una región de grano fino, es la formación de lagunas martensíticas que contribuyen a la fragilización de esta región (7). La fisura se inició a una distancia aproximada de $18 \mathrm{~mm}$ desde los cor-

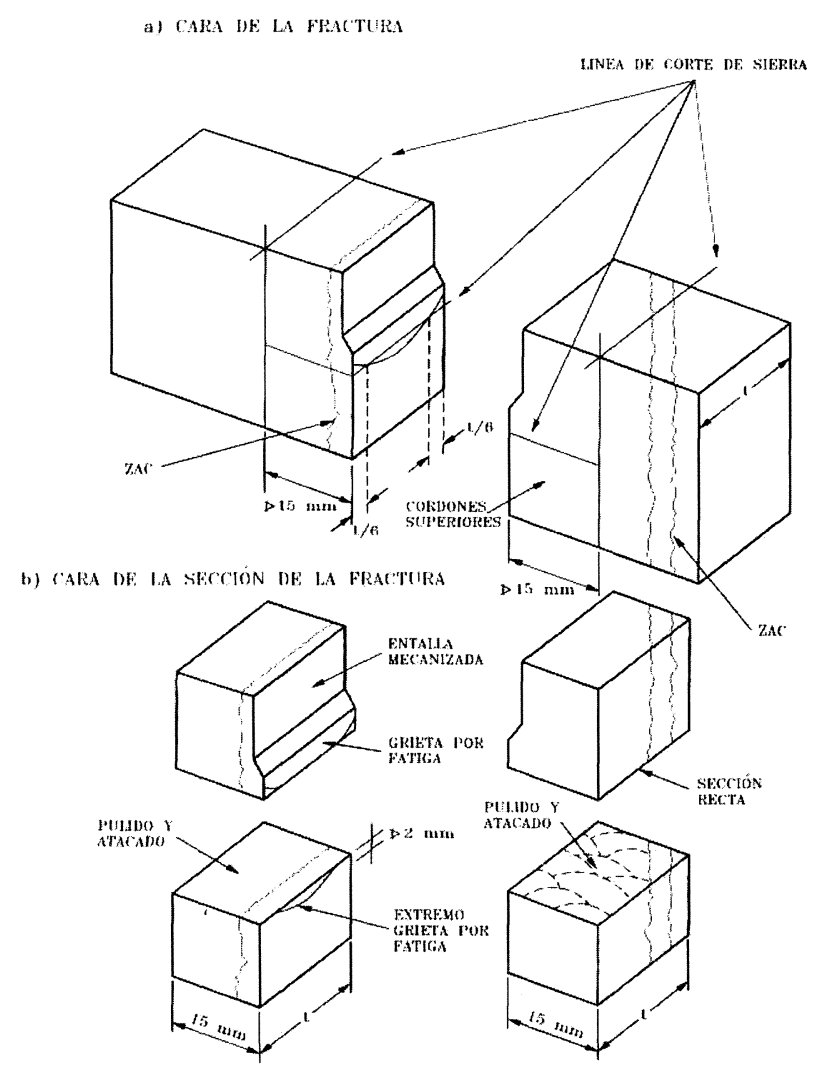

FIG. 9.- Preparación de muestras y seccionado post-ensayo. Estudio fractográfico.

FIG. 9.- Specimen details of post test sectioning through thickness. Fractography.

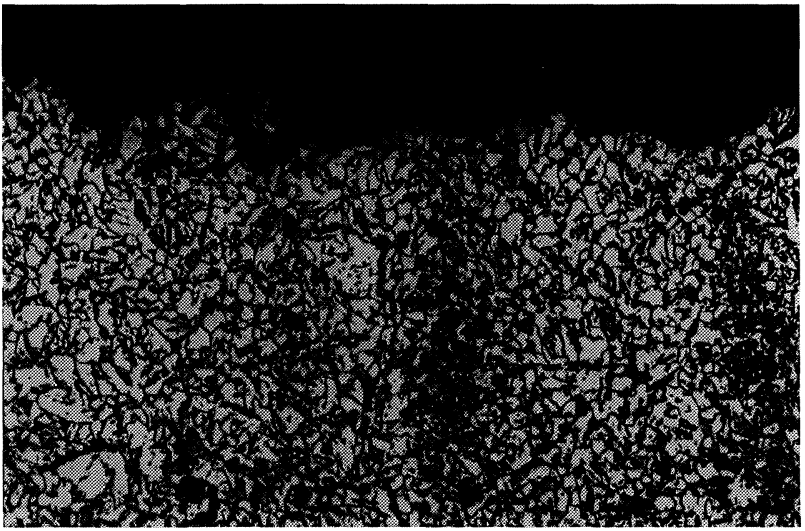

FIG. 10.- Micrografía óptica de la iniciación de la fractura en la probeta P1B. General (iniciación marcada) $\times 50$.

FIG. 10.- Optical micrograph fracture initiation from the specimen P1B. General (initiation marked) $\times 50$.

dones superiores del lado de la $\mathrm{V}$ grande, como se muestra en la figura 13 que, conforme al diagrama de barras de la figura 14 , corresponde a una región de grano mayor de 7 ASTM, región intercrítica. 


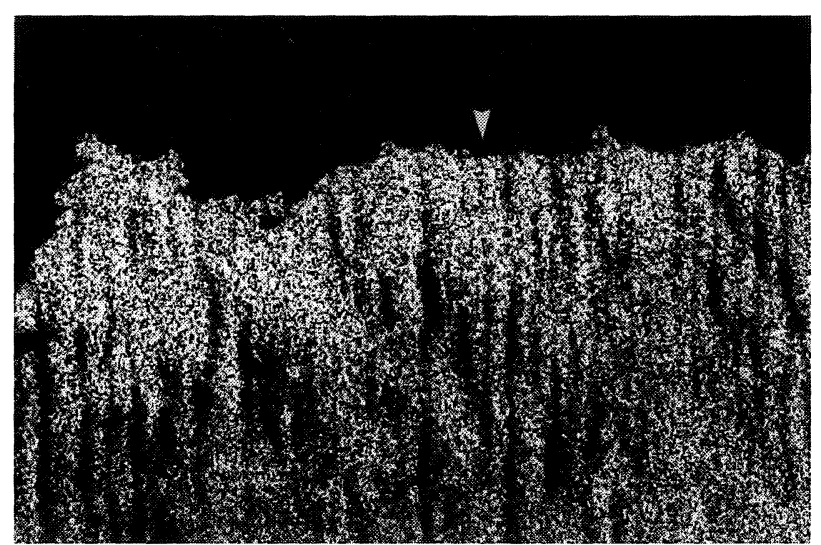

FIG. 11.- Micrografía óptica de la iniciación de la fractura en la probeta P1B. Detalle (iniciación marcada) $\times 320$.

FIG. 11.- Optical micrograph fracture initation from the specimen PIB. Detail (initiation marked) $\times 320$.

Para la probeta P1C, que también demostró fractura frágil, se observa detalle de la sección de comienzo de la grieta (Fig. 15). En las figuras 16 y 17 se puede ver, con mayor detalle, el inicio de la rotu-

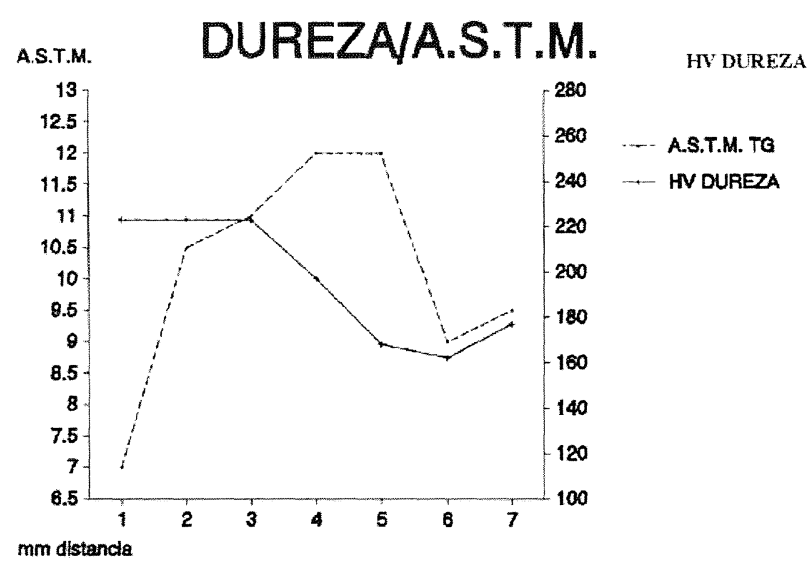

Fig. 12.- Dureza. Tamaño de grano. Distancia a L.F. Probeta P1T.

FIG. 12.-Hardness. Grain size. Distance from F.L. Specimen P1T.

ra en la zona de grano grueso a 2,3 mm de LF (Tabla IV). El diagrama de barras de la figura 14 muestra que la fisura se inicia en la región de metal aportado, a $10 \mathrm{~mm}$ de los cordones que forman la $\mathrm{V}$ grande.

TABLA IV.- Regiones y temperatura de la ZAC en función de la distancia a la L.F.

TABLE IV.-Zones and temperatures in the HAZ from the F.L.

\begin{tabular}{|c|c|c|c|c|c|}
\hline \multirow{2}{*}{$\underset{{ }^{\circ} \mathrm{C}}{\text { Temperatura ZAC }}$} & \multirow{2}{*}{ Región ZAC } & \multicolumn{2}{|c|}{ Proceso SAW } & \multicolumn{2}{|c|}{ Proceso SMAW } \\
\hline & & $\begin{array}{c}\text { Distancia ECS } \\
\mathrm{mm}\end{array}$ & $\begin{array}{l}\text { Distancia L.F. } \\
\mathrm{mm}\end{array}$ & $\begin{array}{c}\text { Distancia ECS } \\
\mathrm{mm}\end{array}$ & $\begin{array}{l}\text { Distancia L.F. } \\
\text { mm }\end{array}$ \\
\hline $1.450<\mathrm{Tp}<1.527$ & Grano grueso & 8,7 & $0<\mathrm{d}<0,7$ & 4,35 & $<\mathrm{d}<0,35$ \\
\hline $1.100<\mathrm{Tp}<1.450$ & Crecimiento de grano & 10,3 & $0,7<\mathrm{d}<2,3$ & 5,69 & $0,3<\mathrm{d}<1,69$ \\
\hline $910<\mathrm{Tp}<1.100$ & Grano fino & 11,3 & $2,3<\mathrm{d}<3,3$ & 6,65 & $1,69<\mathrm{d}<2,65$ \\
\hline $722<\mathrm{Tp}<910$ & Intercrítica & 13 & $3,3<\mathrm{d}<5$ & 8 & $2,65<\mathrm{d}<4$ \\
\hline $600<\mathrm{Tp}<722$ & Subcrítica & 14,5 & $5<d<6,5$ & 9,3 & $<\mathrm{d}<5,3$ \\
\hline 500 & & 16,4 & 8,4 & 10,5 & 6,5 \\
\hline
\end{tabular}

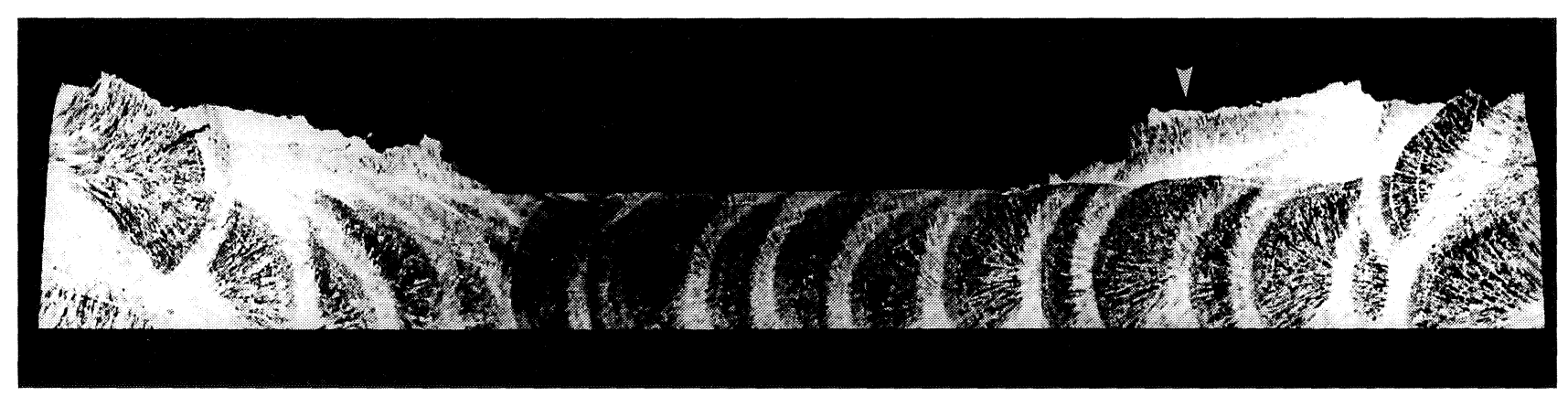

FIG. 13. - Macrografía óptica de la sección transversal por el lado de iniciación de la fractura en la probeta P1B.

FIG. 13.- Optical macrograph of the precrack location section from specimen PIB. 


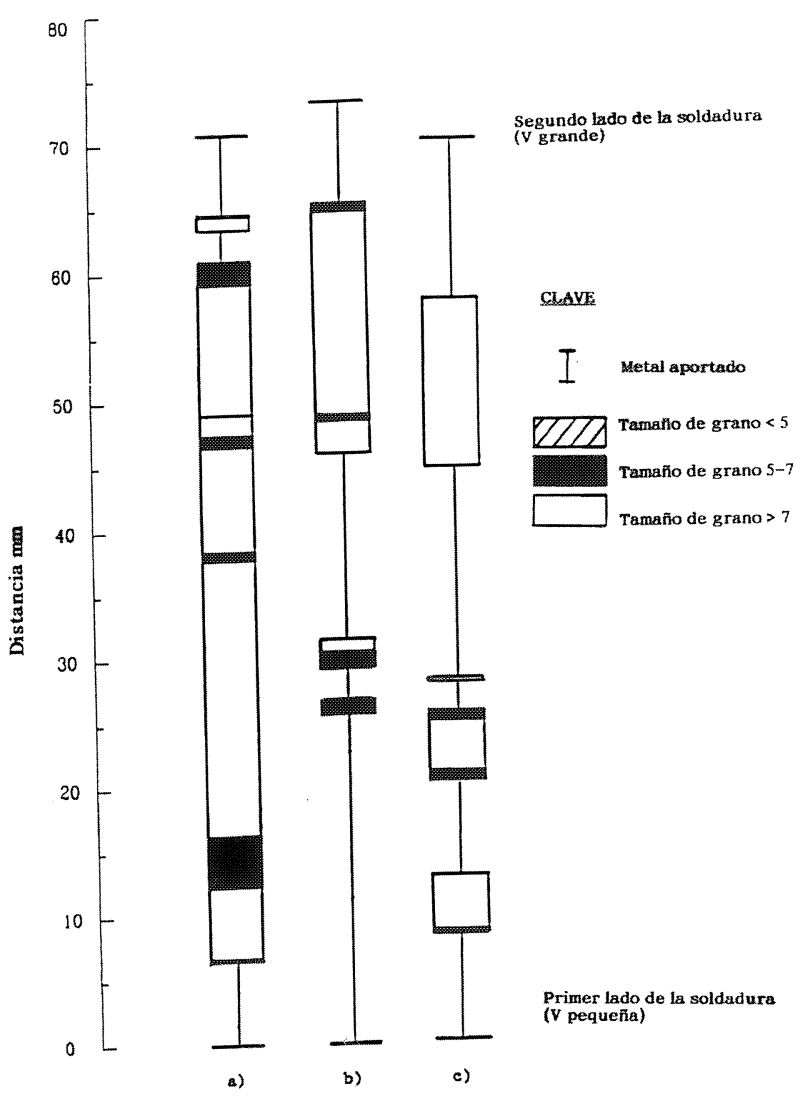

FIG. 14.- Diagrama de barras de la probeta prefisurada localizando varios tipos de microestructura ensayadas: a) P1A; b) P1B; c) P1C.

FIG. 14.- Bar chart locating the various microstructure tested specimens: a) PIA; b) PIB; c) PIC.

Después de ser atacadas con Nital al $2 \%$ y siguiendo la misma técnica utilizada para la valoración del tamaño de grano, desde dichas secciones tomadas por la cara de la fractura se obtuvo una valoración del porcentaje del tamaño de grano de cada

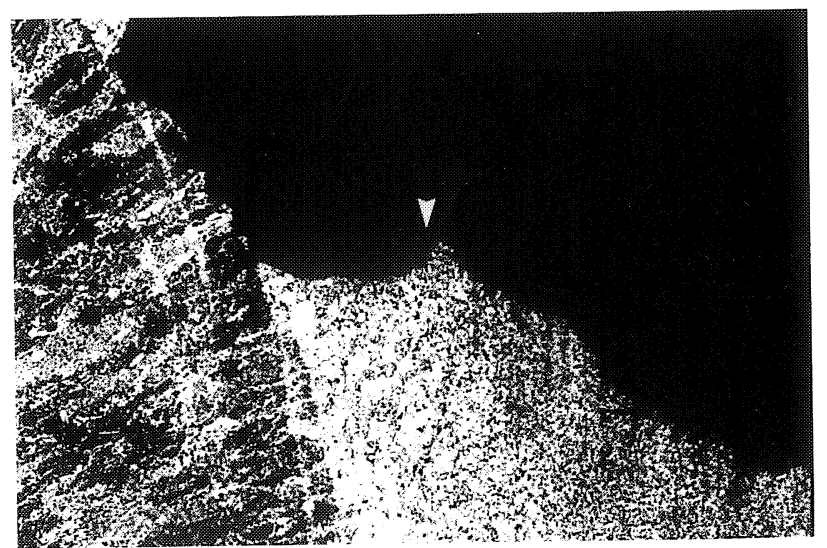

FIG. 16.- Micrografía óptica de la iniciación de la fractura en la probeta P1C. General (iniciación marcada) $\times 50$.

FIG. 16.- Optical micrograph of the fracture initation from specimen PIC. General (initation marked) $\times 50$.

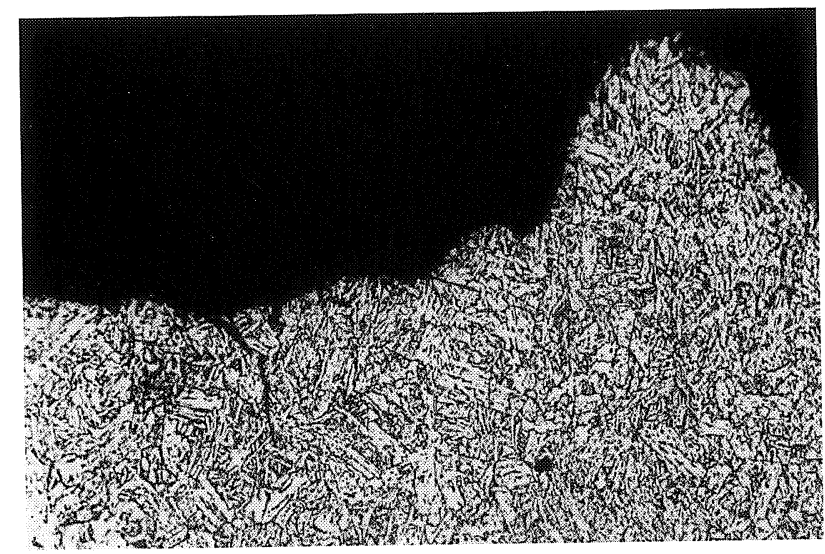

FIG. 17.- Micrografía óptica de la iniciación de la fractura en la probeta P1C. Detalle. $\times 320$.

FIG. 17.- Optical micrograph of the fracture initation from specimen PIC. Detail $\times 320$.

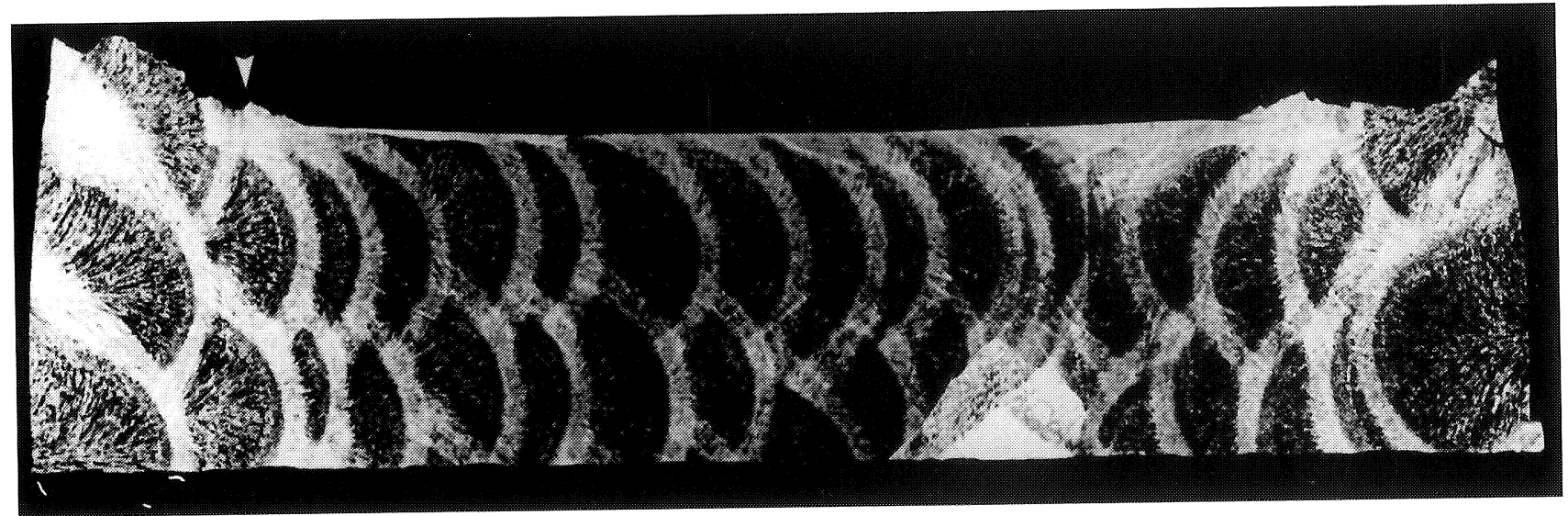

FIG. 15.- Macrografía óptica de la sección transversal por el lado de iniciación de la fractura en la probeta P1C.

FIG. 15.-Optical macrograph of the fracture initation side from specimen PIC. 
una de las zonas evaluadas, como se indica en las tablas I y II.

Una valoración final de la propagación de la grieta, conforme la EEMUA 158 apartado A2.4.4, requiere que la totalidad de la fisura se encuentre dentro de una distancia de $0,5 \mathrm{~mm}$ de $\mathrm{LF}$, dentro de $\operatorname{los} 2 / 3$ del espesor de la probeta.

\subsection{CTOD. Resultados y conclusiones}

Las macrografías (Figs. 18, 19 y 20) muestran la cara de la prefisura de cada probeta, donde por su aspecto se puede diferenciar la fractura dúctil (P1A) de las frágiles (P1B y P1C).

La relación del tamaño de grano, interceptado porcentualmente por la fisura en la ZAC de cada una de las probetas conforme la norma ASTM E 112 (2), se representa en las tablas I y II.

El reparto y la localización de estos porcentajes quedan dibujados en el diagrama de barras (Fig. 14). Como se puede apreciar, las tres probetas muestran un alto porcentaje de grano fino en la ZAC, fruto del control de la energía aportada por la soldadura, $2,4 \mathrm{~kJ} / \mathrm{mm}$.

De las probetas P1B y P1C, que mostraron un comportamiento de fractura frágil, se obtuvo una segunda sección para análisis. Para la sección P1B

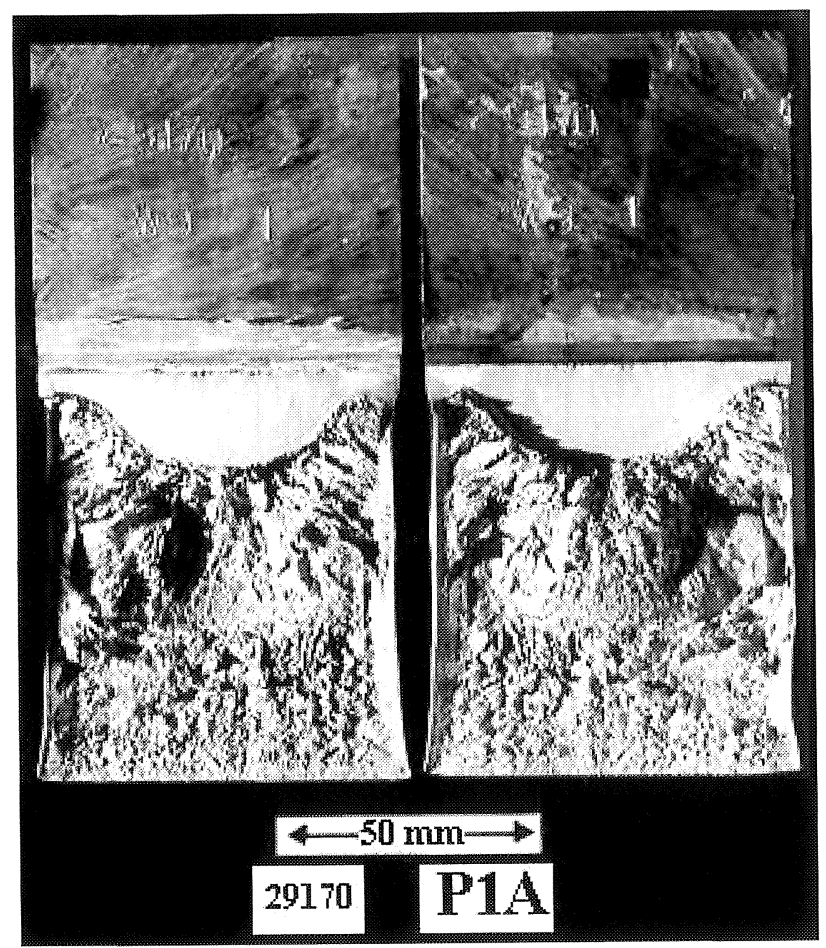

Fig. 18.- Macrografía óptica de la superficie de la fractura de la probeta $\mathrm{P} 1 \mathrm{~A}$.

FIG. 18.- Optical macrograph of the fracture surfaces from specimen PIA.

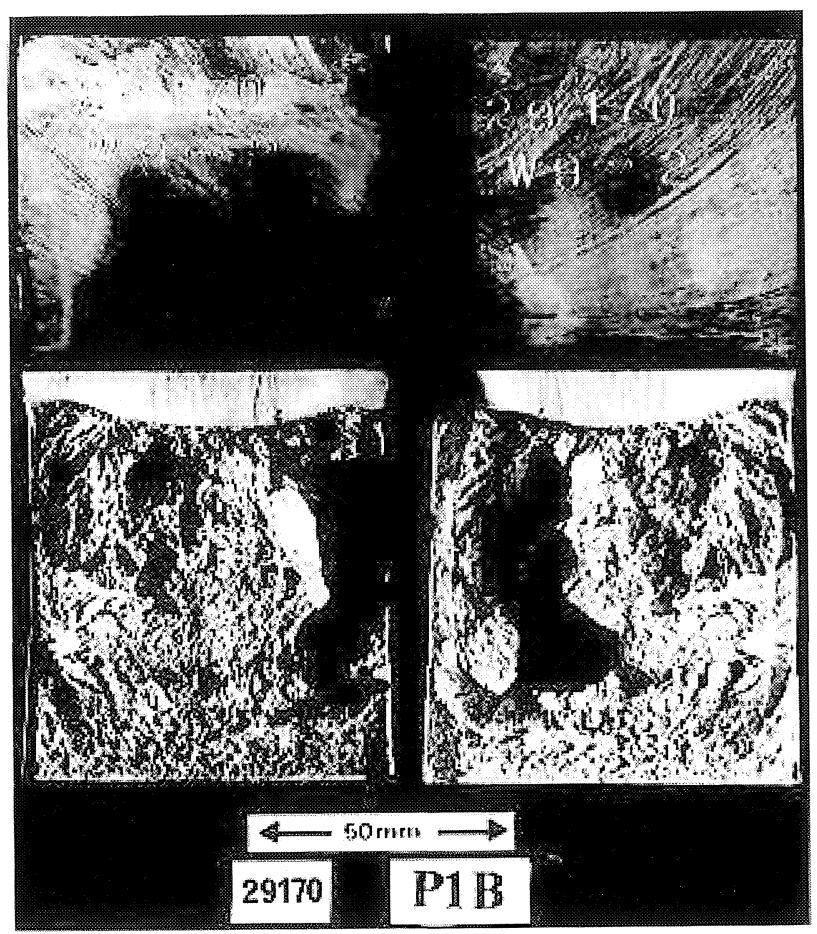

FIG. 19.- Macrografía óptica de la superficie de la fractura de la probeta $\mathrm{P} 1 \mathrm{~B}$.

FIG. 19.- Optical macrograph of the fracture surfaces from specimen PlB.

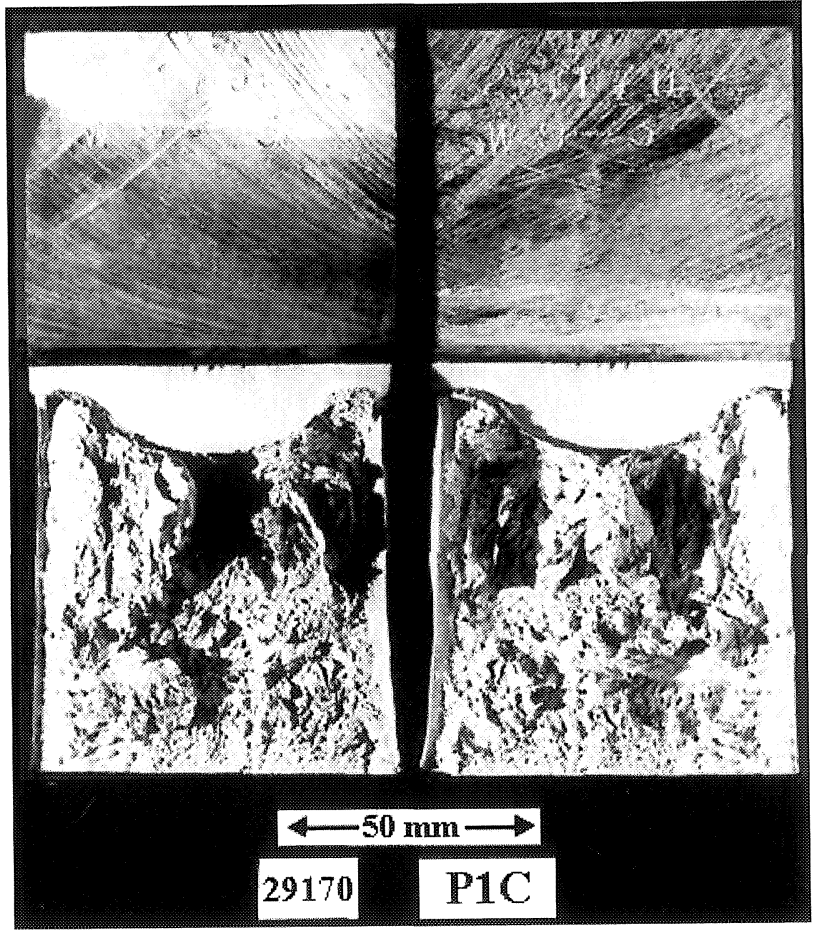

Fig. 20.- Macrografía óptica de la superficie de la fractura de la probeta $\mathrm{P} 1 \mathrm{C}$.

FIG. 20.- Optical macrograph of the fracture surfaces from specimen PIC. 
(Figs. 10, 11 y 13), la fractura se inició a 3,3 mm de LF, en zona intercrítica de grano fino (región intercrítica, IC, tabla IV).

Para la probeta P1C, la macrografía de la sección de iniciación de grieta se da en las figuras 15, 16 y 17. En este caso, la iniciación de la fractura comenzó en la parte más exterior de crecimiento de grano, a 2,3 mm de LF (GC), muy próximo a la región de grano fino (Tabla IV, proceso SAW).

Se midió la cantidad de la grieta por fatiga que se encontraba en los $0,5 \mathrm{~mm}$ de la LF (excluyendo la cantidad de metal de soldadura), conforme a la norma EEMUA 158 apartado A 2.4.4 (4), encontrando las valoraciones dadas en la tabla $\mathrm{V}$.

De los valores especificados en la tabla $\mathrm{V}$, deducimos que ninguna de las grietas se encuentra al $100 \%$ dentro de los $0,5 \mathrm{~mm}$, que requiere dicha norma. Como se observa en ésta, los mayores porcentajes de longitudes de grietas dentro de los 0,5 $\mathrm{mm}$ de LF corresponden a las fracturas frágiles, probetas P1B y P1C.

Las conclusiones obtenidas en los ensayos de mecánica de fractura sobre las probetas del panel CTOD 1-1G HAZ, conforme a la referida norma EEMUA, apartado 2.4 .4 y a la BS $5762 \mathrm{a}-10{ }^{\circ} \mathrm{C}$, en un proceso de soldadura S.A.W., fueron (Tabla $\mathrm{VI})$ :

TABLA V.- Porcentajes de grieta muestreada dentro de los 0,5 mm de la L.F.

TABLE V. - Percentages of fracture tested within 0.5 $\mathrm{mm}$ from F.L.

\begin{tabular}{|c|c|}
\hline Probeta & $\begin{array}{c}\text { Porcentaje de longitud de grieta } \\
\text { dentro de los } 0,5 \mathrm{~mm} \text { de L.F. }\end{array}$ \\
\hline P1A & $11 \%$ \\
P1B & $48 \%$ \\
P1C & $31 \%$ \\
\hline
\end{tabular}

TABLA VI.- Sumario de los resultados de los ensayos CTOD de la ZAC a $-10{ }^{\circ} \mathrm{C}$ en panel CTOD 1-1G HAZ.

TABLE VI.- Summary of HAZ CTOD test results at $-10{ }^{\circ} \mathrm{C}$ in the panel CTOD $1-1 \mathrm{G} \mathrm{HAZ}$.

\begin{tabular}{|c|c|c|}
\hline Probeta* & CTOD $(\mathrm{mm})$ & $\begin{array}{c}\text { Tipo de resultado } \\
\text { para BS 5762 }\end{array}$ \\
\hline P1A & 1,80 & $\delta$, ensayo parado * \\
P1B & 1,31 & $\delta_{\mathrm{u}} *$ \\
P1C & 1,58 & $\delta_{\mathrm{u}} *$ \\
\hline
\end{tabular}

* Todas las probetas son $\mathrm{B} \times 2 \mathrm{~B}$ entalladas
- Los ensayos CTOD fueron válidos conforme a la norma BS 5762.

- Los valores de CTOD encontrados $\delta=1,8 \mathrm{~mm}$, $\delta_{\mathrm{u}}=1,31 \mathrm{~mm}, \delta_{\mathrm{u}}=1,58 \mathrm{~mm}$ excedieron sobradamente el valor mínimo requerido $\delta=0,2 \mathrm{~mm}$

- Ninguno de los ensayos cumplieron el requerimiento de la norma EEMUA 158 (4), en lo referente a que la totalidad de la longitud de la grieta debe estar dentro de los $0,5 \mathrm{~mm}$ de la LF (región de grano grueso).

- En la figura 21 podemos observar que, a mayores valores de CTOD se produce un valor más alto de crecimiento estable de grieta, como corresponde a una estructura más tenaz.

- La zona intercrítica mostró fragilidad, iniciándose la fractura por esta región de grano fino.

\subsection{Resiliencia. Resultados y conclusiones}

Las probetas, de dimensiones $55 \mathrm{~mm} \times 10 \mathrm{~mm} \times 10$ $\mathrm{mm}$ y entalla de $2 \mathrm{~mm}$, fueron tomadas de los puntos que se indican en la figura 22 , tanto sin tratamiento térmico post-soldeo como con dicho tratamiento.

Los resultados que han sido llevados al gráfico permiten establecer las siguientes observaciones:

- La resiliencia aumenta con tratamiento térmico post-soldeo.

- La resiliencia disminuye considerablemente en la LF (Fig. 23).

- A medida que existe un alejamiento de LF, como vemos a $5 \mathrm{~mm}$, la resiliencia aumenta, llegando a bajar a $90 \mathrm{~J}$ en la raíz, situándola al límite de lo admisible, debido a la fragilidad que le proporciona la zona de grano grueso.

- La resiliencia mínima, $90 \mathrm{~J}$, se encuentra en la LF de los cordones superiores.

- Sin tratamiento térmico post-soldeo y temperatura de $-40{ }^{\circ} \mathrm{C}$ en $\mathrm{LF}$, la resiliencia en raíz es

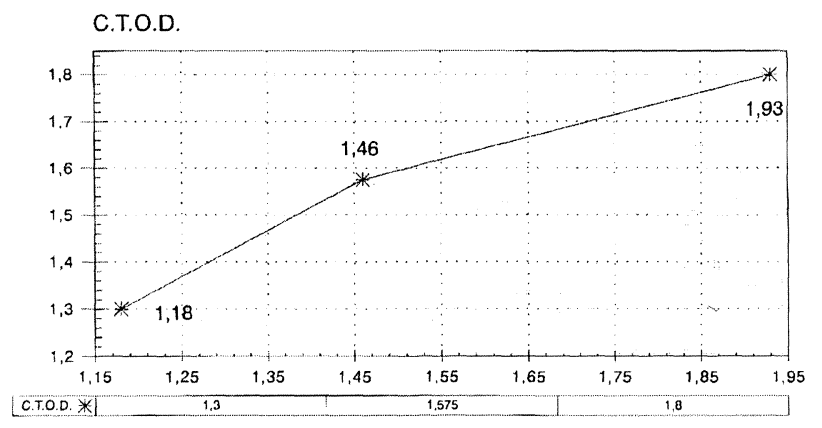

FIG. 21.- CTOD. Crecimiento estable. Proceso SAW.

FIG. 21.-Stable crack extension. SAW process. 

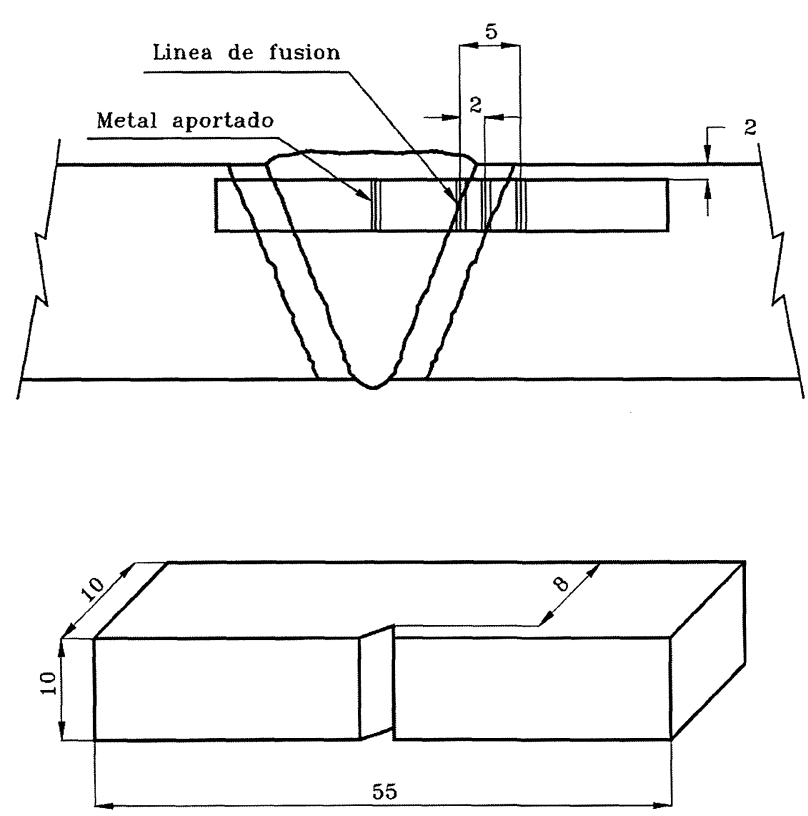

FIG. 22.- Probeta para ensayo resiliencia péndulo de Charpy y su localización.

FIG. 22.- Charpy specimen piece and its location.

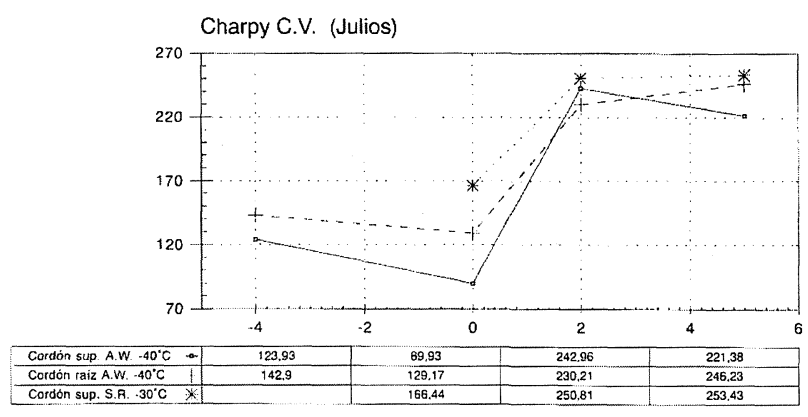

FIG. 23.- Resiliencia. Distancia a L.F.

FIG. 23.-Charpy results. Distance from F.L.

mayor que en los cordones superiores. Para los puntos que se encuentran a $2 \mathrm{~mm}$ de LF, la resiliencia es mayor en los cordones superiores que en la raíz, en parte debido al tratamiento térmico que supone el efecto multipasada para los cordones superiores y en parte a una disminución de la tenacidad por envejecimiento en la raíz. Esta diferencia se hace menos acusada, conforme se produce un alejamiento de LF hacia el metal base.

Como era de esperar, en LF la resiliencia disminuye, tomando los mínimos valores, ya que como se ha visto, el grano grueso es más frágil y corresponde a una estructura entre cuyos productos de transformación se encuentra la martensítica.

\subsection{Dureza. Resultados y conclusiones}

Se comprueba una mayor dureza desde LF a la región de grano grueso. También, como se ha visto en los ensayos de fractura CTOD, la iniciación de la grieta tiene lugar entre los 2 y $3 \mathrm{~mm}$ de LF, incumpliendo la norma que pide que el $100 \%$ de la longitud de la grieta por fatiga esté dentro de los $0,5 \mathrm{~mm}$ de LF, región de grano grueso.

Los ensayos de dureza, llevados a cabo mediante HV Vicker 10 en la probeta P1S, quedan reflejados en la gráfica de la figura 24 .

Se observa que la mayor dureza está localizada en el metal depositado en la raíz, con 214,48 HV10, que se corresponde con la menor resiliencia y fractura encontrados en la probeta. Esto era de esperar ya que en esta zona se encuentra el mayor tamaño de grano y la mayor fragilidad, lo que incide en una mayor dureza. En la gráfica de dureza (Fig. 24) se observa un paralelismo entre la dureza de los cordones superiores, raíz e inferiores. De los valores obténidos en el ensayo CTOD en relación con el crecimiento estable se deduce una proporcionalidad entre ambos, obteniéndose casi una recta, como corresponde a los valores esperados entre ambos parámetros. Así mismo, se observa un crecimiento constante y positivo, es decir, a un mayor crecimiento estable, mayor valor de CTOD (gráfico de la figura 21). En el ensayo de dureza HV10 llevado a cabo sobre la probeta se obtuvieron valores razonablemente parecidos en tres secciones de su espesor, correspondiendo el valor más alto a la raíz. De la observación de estos valores se deduce que el perfil de la gráfica obtenida HV10/(distancia L.F.) corresponde al esperado para un acero templado y revenido, teniendo una dureza elevada en el W.M. y zonas próximas a la LF, para ir disminuyendo a consecuencia del recocido que sufre el material por efecto de las continuas pasadas, y terminando en una dureza, que aún siendo superior a la zona de recocido, es inferior a la del W.M.

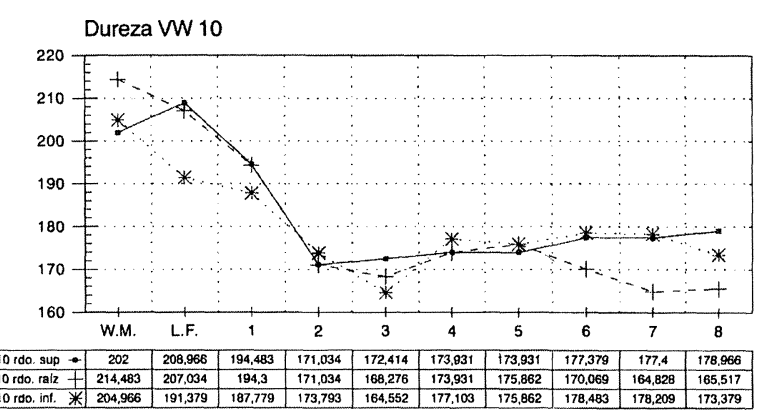

FIG. 24.- Dureza. Distancia a L.F.

FIG. 24.-Hardness. Distance from F.L. 
Si comparamos estos valores de dureza con los de resiliencia obtenidos en el péndulo de Charpy, en función a la distancia de LF, deducimos que los puntos de inflexión de mayor dureza corresponden a la menor resiliencia y viceversa (Fig. 25). En este gráfico dureza/resiliencia se han representado los valores obtenidos en la probeta que estamos probando en los cordones superiores y de raíz, resultando, como puede verse, una aproximación entre los valores obtenidos en la raíz y los obtenidos en los cordones superiores.

No se observa una significativa disminución de la resiliencia en la región intercrítica.

La mayor dureza se obtiene en el W.M. y LF, así como zonas más próximas a dicha L.F., disminu-

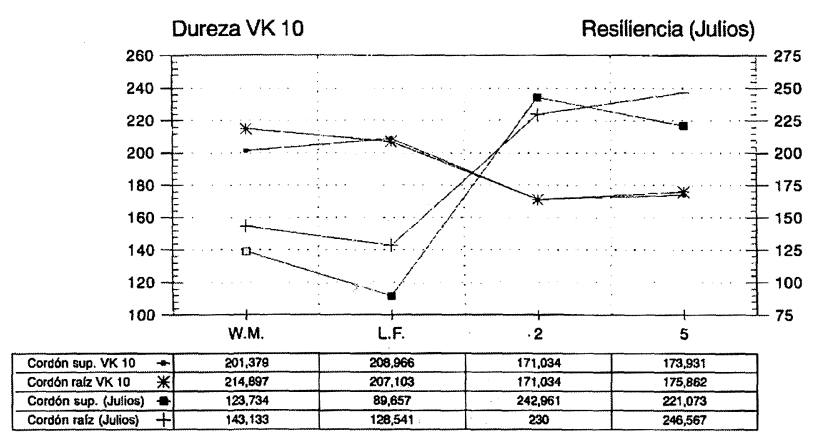

FIG. 25.- Dureza. Resiliencia. Distancia a L.F.

FIG. 25.- Hardness. Charpy results. Distance from F.L. yendo conforme nos alejamos de la zona de mayor calentamiento.

También se puede observar que la raíz muestra una ligera mayor dureza que los cordones superiores, debido al ciclo térmico sufrido en esa zona que le da mayor fragilidad. Como consecuencia de lo anterior, los cordones superiores son los que muestran una mayor resiliencia.

\section{REFERENCIAS}

(1) Grong, O. M. Akselsen Institute of Technology of Trondheim. "Microestructure and toughness in haz of boron containing steels". Sintef report STF 34 A84065, 1984.

(2) ASTME 112-88. Method for determining average grain size. American Society for Testing and Material, 1988: 1290.

(3) British Standards Institution. BS 5762. 1979. Method for crack opening displacement (C.O.D.) testing.

(4) The Engineering Equipment and Material Users Association. Constructions specification for fixed off-shore structures in the North Sea. Publication 158.

(5) IRWIN GR. Linear fracture mechanics, fracture transition, and fracture control. Eng. Fract. Mech., 2, (1), 1968: 241257.

(6) ROBERT C. y Newton C. Interpretive report in small scale test correlations with KI C Datas. Weld. Res. Cound. Bull., 265, 1983.

(7) Gonzalez, R.E. Tesis Doctoral. Estudio de tenacidad a la fractura relacionada con la microestructura en la zona afectada por el calor (ZAC) de los aceros microaleados para las plataformas Off Shore. Facultad de Ciencias de la Universidad de Cádiz. 1994. 